\title{
High\#Redshift Galaxies: Their Predicted Size and Surface Brightness Distributions and Their Gravitational Lensing Probability
}

\section{Citation}

Barkana, Rennan, and Abraham Loeb. 2000. “High\#Redshift Galaxies: Their Predicted Size and Surface Brightness Distributions and Their Gravitational Lensing Probability." The Astrophysical Journal 531 (2): 613-23. https://doi.org/10.1086/308503.

\section{Permanent link}

http://nrs.harvard.edu/urn-3:HUL.InstRepos:41393262

\section{Terms of Use}

This article was downloaded from Harvard University's DASH repository, and is made available under the terms and conditions applicable to Other Posted Material, as set forth at http:// nrs.harvard.edu/urn-3:HUL.InstRepos:dash.current.terms-of-use\#LAA

\section{Share Your Story}

The Harvard community has made this article openly available. Please share how this access benefits you. Submit a story. 
The Astrophysical Journal, 531:613-623, 2000 March 10

(c) 2000. The American Astronomical Society. All rights reserved. Printed in U.S.A.

\title{
HIGH-REDSHIFT GALAXIES: THEIR PREDICTED SIZE AND SURFACE BRIGHTNESS DISTRIBUTIONS AND THEIR GRAVITATIONAL LENSING PROBABILITY
}

\author{
RENNAN BARKANA \\ Institute for Advanced Study, Olden Lane, Princeton, NJ 08540; barkana@ias.edu
}

AND

ABRAHAM LOEB

Astronomy Department, Harvard University, 60 Garden Street, Cambridge, MA 02138; aloeb@cfa.harvard.edu Received 1999 June 22; accepted 1999 October 26

\begin{abstract}
Direct observations of the first generation of luminous objects will likely become feasible over the next decade. The advent of the Next Generation Space Telescope (NGST) will allow imaging of numerous galaxies and mini-quasars at redshifts $z \gtrsim 5$. We apply semianalytic models of structure formation to estimate the rate of multiple imaging of these sources by intervening gravitational lenses. Popular cold dark matter $(\mathrm{CDM})$ models for galaxy formation yield a lensing optical depth of $\sim 1 \%$ for sources at $z \sim 10$. The expected slope of the luminosity function of the early sources implies an additional magnification bias of $\sim 5$, bringing the fraction of lensed sources at $z=10$ to $\sim 5 \%$. We estimate the angular size distribution of high-redshift disk galaxies and find that most of them are more extended than the resolution limit of $N G S T, \sim 00^{\prime \prime} 06$. We also show that there is only a modest redshift evolution in the observed mean surface brightness of galaxies at $z \gtrsim 2$. The expected increase by $1-2$ orders of magnitude in the number of resolved sources on the sky, due to observations with NGST, will dramatically improve upon the statistical significance of existing weak-lensing measurements. We show that despite this increase in the density of sources, confusion noise from $z>2$ galaxies is expected to be small for NGST observations.
\end{abstract}

Subject headings: cosmology: theory — galaxies: formation — galaxies: fundamental parameters gravitational lensing

\section{INTRODUCTION}

Current observations reveal the existence of galaxies out to redshifts as high as $z \sim 6.7$ (Chen, Lanzetta, \& Pascarelle 1999; Weymann et al. 1998; Dey et al. 1998; Spinrad et al. 1998; Hu, Cowie, \& McMahon 1998) and bright quasars out to $z \sim 5$ (Fan et al. 1999). Based on sources for which high-resolution spectra are available, the intergalactic medium appears to be predominantly ionized at this epoch, implying the existence of ionizing sources at even higher redshifts (Madau 1999; Madau, Haardt, \& Rees 1999; Haiman \& Loeb 1998, 1999; Gnedin \& Ostriker 1997).

Hierarchical cold dark matter (CDM) models for structure formation predict that the first baryonic objects appeared near the Jeans mass $\left(\sim 10^{5} M_{\odot}\right)$ at redshifts as high as $z \sim 30$ (Haiman \& Loeb 1999, and references therein). The Next Generation Space Telescope (NGST), planned for launch in 2008, is expected to reach an imaging sensitivity of better than $1 \mathrm{nJy}$ in the infrared, which will allow it to detect galaxies or mini-quasars at $z \gtrsim 10$.

In this paper we explore the ability of $N G S T$ to extend gravitational lensing studies well beyond their current limits. Because of the increased path length along the line of sight to the most distant sources, their probability for being lensed is expected to be the highest among all possible sources. Sources at $z>10$ will often be lensed by $z>2$ galaxies, whose masses can then be determined with lens modeling. Similarly, the shape distortions (or weak lensing) caused by foreground clusters of galaxies will be used to determine the mass distributions of less massive and higher redshift clusters than currently feasible. In addition to studying the lensing objects, observers will exploit the magnification of the sources to resolve and study more distant galaxies than would otherwise be possible.
The probability for strong gravitational lensing depends on the abundance of lenses, their mass profiles, and the angular diameter distances among the source, the lens, and the observer. The statistics of existing lens surveys have been used at low redshifts to constrain the cosmological constant (for the most detailed work, see Kochanek 1996a and references therein), although substantial uncertainties remain regarding the luminosity function of early-type galaxies and their dark matter content (Cheng \& Krauss 1999; Chiba \& Yoshii 1999). The properties of dark matter halos will be better probed in the future by individual as well as statistical studies of the large samples of lenses expected from quasar surveys such as the 2-Degree Field (Croom et al. 1999) and the Sloan Digital Sky Survey. ${ }^{1}$ Given the early stage of observations of the redshift evolution of galaxies and their dark halos, we adopt a theoretical approach in our analysis and use the abundance of dark matter halos as predicted by the Press-Schechter (1974, hereafter PS) model. A similar approach has been used previously for calculating lensing statistics at low redshifts, with an emphasis on lenses with image separations above 5" (Narayan \& White 1988; Kochanek 1995; Nakamura \& Suto 1997) or on lensing rates of supernovae (Porciani \& Madau 2000).

Even when multiple images are not produced, the shape distortions caused by weak lensing can be used to determine the lensing mass distribution. Large numbers of sources are required in order to average away the noise due to the intrinsic ellipticities of sources, and so the mass distribution can only be determined for the extended halos of rich clusters of galaxies (e.g., Hoekstra et al. 1998; Luppino \& Kaiser 1997; Seitz et al. 1996) or statistically for galaxies

\footnotetext{
1 The Sloan Digital Sky Survey (SDSS Collaboration 1996) is available at: http://www.astro.princeton.edu/BBOOK/.
} 
(e.g., Brainerd, Blandford, \& Smail 1996; Hudson et al. 1998). Schneider \& Kneib (1998) have noted that the ability of NGST to take deeper exposures than is possible with current instruments will increase the observed density of sources on the sky, particularly those at high redshifts. The large increase might allow such applications as a detailed weak-lensing mapping of substructure in clusters. Obviously, the source galaxies must be well resolved to allow an accurate shape measurement. Unfortunately, the characteristic galaxy size is expected to decrease with redshift for two reasons: (1) the mean density of collapsed objects scales as the density of the universe at the collapse redshift, namely, as $(1+z)^{3}$; hence, objects of a given mass are expected to be more compact at high redshifts; and (2) the characteristic mass of collapsed objects decreases with increasing redshift in the bottom-up CDM models of structure formation. In the following discussion, we attempt to calculate the size distribution of high-redshift sources. Aside from the obvious implications for weak-lensing studies, the finite size of sources also has important implications for their detectability with NGST above the background noise of the sky brightness.

The outline of the paper is as follows. In $\S 2$ we employ the PS halo abundance in several hierarchical models of structure formation to estimate the lensing rate of the highredshift objects that will be observed with NGST. This lensing rate has been calculated by Marri \& Ferrara (1998), assuming point-mass lenses. We use the simple but more realistic model of a singular isothermal sphere (SIS) profile for dark matter halos and obtain a substantially lower lensing rate. The formation of galactic disks and the distributions of their various properties have been studied by Dalcanton, Spergel, \& Summers (1997; hereafter DSS) and Mo, Mao, \& White (1998; hereafter MMW) in the framework of hierarchical models of structure formation. In $\S 3$ we apply their models to high-redshift sources and find the angular size distribution of galactic disks as a function of redshift. We use this distribution to predict whether observations with NGST will be significantly limited by confusion noise. We also calculate the redshift evolution of the mean surface brightness of disks. Finally, $\S 4$ summarizes the implications of our results.

\section{LENSING RATE OF HIGH-REDSHIFT SOURCES}

\subsection{Calculation Method}

We calculate the abundance of lenses based on the PS halo mass function. Relevant expressions for various CDM cosmologies are given in, e.g., Navarro, Frenk, \& White (1997, hereafter NFW). The PS abundance agrees with $\mathrm{N}$-body simulations on the mass scale of galaxy clusters, but may overpredict the abundance of galaxy halos at present by a factor of 1.5-2 (e.g., Gross et al. 1998). At higher redshifts, the characteristic mass scale of collapsed objects drops, and the PS abundance becomes more accurate for the galaxy-size halos that dominate the lensing rate.

The probability for producing multiple images of a source at a redshift $z_{S}$ due to gravitational lensing by SIS lenses is obtained by integrating over lens redshift $z_{L}$ the differential optical depth (Turner, Ostriker, \& Gott 1984; Fukugita et al. 1992; Peebles 1993, p. 336),

$$
d \tau=16 \pi^{3} n\left(\frac{\sigma}{c}\right)^{4}\left(1+z_{L}\right)^{3}\left(\frac{D_{O L} D_{L S}}{D_{O S}}\right)^{2} \frac{c d t}{d z_{L}} d z_{L},
$$

in terms of the comoving density of lenses $n$, velocity dispersion $\sigma$, look-back time $t$, and angular diameter distances $D$ among the observer, lens, and source. More generally, we replace $n \sigma^{4}$ by

$$
\left\langle n \sigma^{4}\right\rangle=\int \frac{d n\left(M, z_{L}\right)}{d M} \sigma^{4}\left(M, z_{L}\right) d M,
$$

where $d n / d M$ is the PS halo mass function. We assume that $\sigma(M, z)=V_{c}(M, z) / \sqrt{2}$, and we calculate the circular velocity $V_{c}(M, z)$ corresponding to a halo of a given mass as in NFW, except that we vary the virialization overdensity using the fitting formula of Bryan \& Norman (1998). The lensing rate depends on a combination of redshift factors, as well as the evolution of halo abundance. At higher redshifts, halos of a given mass are more concentrated and have a higher $\sigma$, but lower mass halos contain most of the mass in the universe.

When calculating the angular diameter distances, we assume the standard distance formulas in a homogeneous universe. Inhomogeneities, however, cause a dispersion around the mean distance. The non-Gaussian, skewed distribution of distances in hierarchical models is best studied with numerical simulations (e.g., Wambsganss, Cen, \& Ostriker 1998), and can in principle be included selfconsistently in more elaborate calculations of the lensing statistics.

We consider cosmological models with various values of the cosmological density parameters of matter and vacuum (cosmological constant), $\Omega_{0}$ and $\Omega_{\Lambda}$. In particular, we show results for $\Lambda \mathrm{CDM}$ (with $\Omega_{0}=0.3$ and $\Omega_{\Lambda}=0.7$ ), OCDM (with $\Omega_{0}=0.3$ and $\Omega_{\Lambda}=0$ ), and SCDM (with $\Omega_{0}=1$ and $\Omega_{\Lambda}=0$ ). The models assume a Hubble constant $h=0.5$ if $\Omega_{0}=1$ and $h=0.7$ otherwise (where $H_{0}=100 h \mathrm{~km} \mathrm{~s}^{-1}$ $\left.\mathrm{Mpc}^{-1}\right)$. They also assume a primordial scale-invariant $(n=1)$ power spectrum, normalized to the present cluster abundance, $\sigma_{8}=0.5 \Omega_{0}^{-0.5}$ (e.g., Pen 1998 and references therein), where $\sigma_{8}$ is the root mean square (rms) amplitude of mass fluctuations in spheres of radius $8 h^{-1} \mathrm{Mpc}$.

\subsection{Numerical Results}

In Figure 1 we show the variation of the lensing optical depth with source redshift. This plot does not include the magnification bias, which we discuss below. In order to show the relative variation, we normalize each curve to unity at $z_{S}=2$. The dashed curves show results for nonevolving lenses with $\left\langle n \sigma^{4}\right\rangle=$ const in $\Lambda \mathrm{CDM}$, OCDM, and SCDM, from top to bottom. The higher values obtained in low- $\Omega$ models are due to the increased spatial volume in these cosmologies. The solid curves show the results for the PS halo distribution in OCDM, $\Lambda$ CDM, and SCDM, from top to bottom. High redshifts are characterized by a decrease in $d n / d M$ at high masses and an increase at low masses, so that the typical mass of collapsing objects decreases. In the OCDM model, the evolution of $d n / d M$ toward lower masses is slow enough that $\left\langle n \sigma^{4}\right\rangle$ increases with $z$ up to $z \sim 3.5$, which increases the lensing optical depth above the value expected for nonevolving lenses.

For a given source redshift, the distribution of lens redshifts is proportional to $d \tau / d z_{L}$, which is given by equations (1) and (2). In Figure 2 we show the probability density $p\left(z_{L}\right)$, defined so that the fraction of lenses between $z_{L}$ and $z_{L}+d z_{L}$ is $p\left(z_{L}\right) d z_{L}$. We assume PS halos in $\Lambda$ CDM (Fig. 2, solid curves), OCDM (dashed curves), or SCDM (dotted 


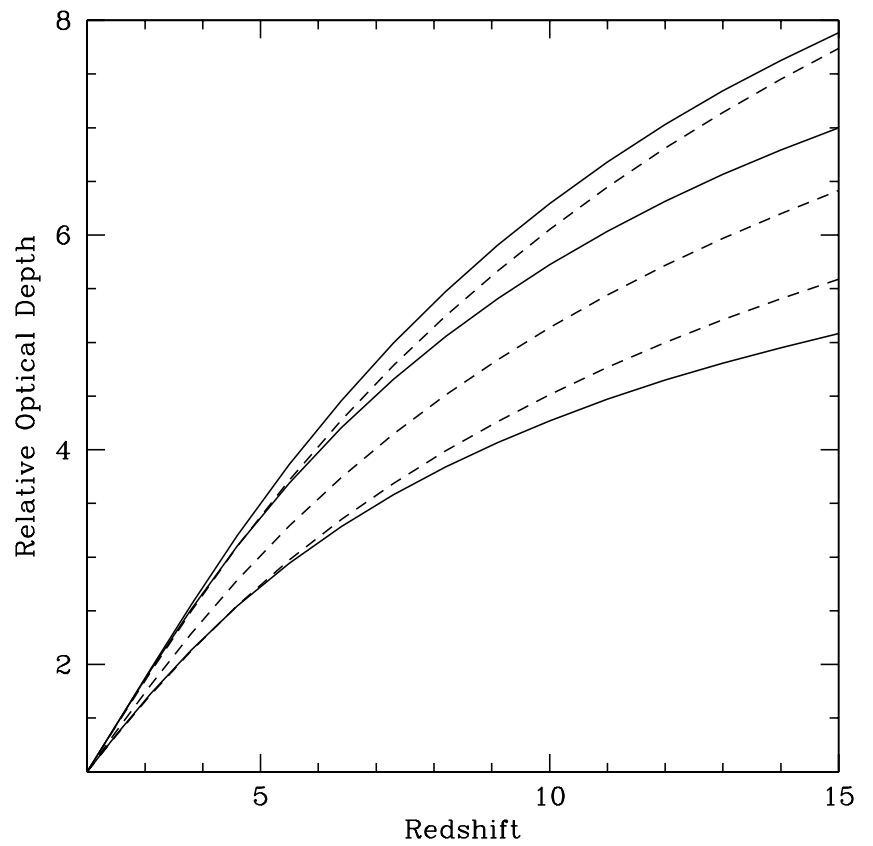

FIG. 1.- Relative variation of the lensing optical depth with source redshift (magnification bias not included), with each curve normalized to unity at $z_{S}=2$. We plot the results for PS halos (solid curves) in OCDM, $\Lambda \mathrm{CDM}$, and SCDM, from top to bottom. These are compared to the corresponding results for nonevolving lenses (dashed curves) in $\Lambda \mathrm{CDM}$, OCDM, and SCDM, from top to bottom.

curves). In each cosmological model, we consider a source at $z_{S}=5$ or 10 , where the higher curve at $z_{L}<1$ corresponds to $z_{S}=5$. The curves peak around $z_{L}=1$ in the low- $\Omega$ models and around $z_{L}=0.7$ in SCDM. In each case, a significant fraction of the lenses are above redshift $2: 20 \%$ for

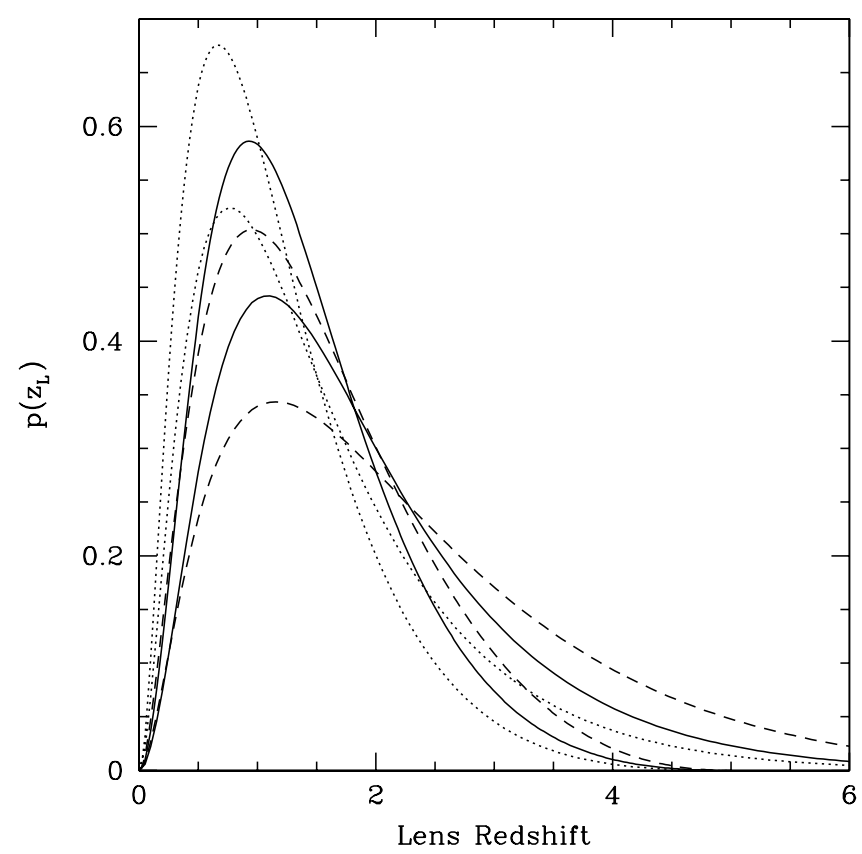

FIG. 2.-Distribution of lens redshifts for a fixed source redshift, for PS halos in $\Lambda$ CDM (solid curves), OCDM (dashed curves), and SCDM (dotted curves). In each model, curves are shown for a source at $z_{S}=5$ and for $z_{S}=10$, where the higher curve at $z_{L}<1$ corresponds to $z_{S}=5$. The probability density $p\left(z_{L}\right)$ is shown, where the fraction of lenses between $z_{L}$ and $z_{L}+d z_{L}$ is $p\left(z_{L}\right) d z_{L}$.
$z_{S}=5$ and $36 \%$ for $z_{S}=10$ in $\Lambda$ CDM. The $z_{L}>2$ fractions are higher in OCDM (26\% for $z_{S}=5$ and $48 \%$ for $\left.z_{S}=10\right)$ and lower in SCDM (13\% for $z_{S}=5$ and $26 \%$ for $\left.z_{S}=10\right)$.

The fraction of lensed sources in an actual survey is enhanced, relative to the above lensing probability, by the so-called magnification bias. At a given observed flux level, unlensed sources compete with lensed sources that are intrinsically fainter. Since fainter galaxies are more numerous, the fraction of lenses in an observed sample is larger than the optical depth discussed above. The magnification bias is calculated in detail below, but for the purpose of the discussion here, we adopt a uniform enhancement factor of 5 when computing the lensing fraction. Our results for the different cosmological models are summarized in Table 1. At $z_{S}=2$, we compare the results from the hierarchical PS models to a no-evolution model of the lens population based on the local luminosity function of galaxies. The last column of Table 1 shows the results (with a magnification bias factor of 5), for example, for the parameters of the no-evolution model of Kochanek (1996a), who adopted a number density of $n_{e}=6.1 h^{3} \times 10^{-3} \mathrm{Mpc}^{-3}$ of E/S0 galaxies, a Schechter function slope $\alpha=-1$, a Faber-Jackson exponent $\gamma=4$, and a characteristic dark matter velocity dispersion of $\sigma_{*}=225 \mathrm{~km} \mathrm{~s}^{-1}$. The PS models yield a higher lensing fraction, although the difference is small for the $\Lambda C D M$ model. In all the PS models, the fraction of multiply imaged systems at $z_{S}=10$ is around $5 \%$ if the magnification bias is 5 .

In the SIS model, the two images of a multiply imaged source have a fixed angular separation, independent of source position, of $\Delta \theta=8 \pi(\sigma / c)^{2}\left(D_{L S} / D_{O S}\right)$. The overall distribution of angular separations is shown in Figure 3 for $\Lambda \mathrm{CDM}$ (solid curves), OCDM (dashed curves), and SCDM (dotted curves). The results are illustrated for $z_{S}=2,5$, and 10 in each model. Image separations are typically reduced by a factor of 2-3 between $z_{S}=2$ and $z_{S}=10$, almost entirely due to the evolution of the lenses. With the NGST resolution of $\sim 0$ ".06, a large majority $(\sim 85 \%)$ of lenses with $\Delta \theta<5^{\prime \prime}$ can be resolved even for $z_{S}=10$. Note, however, that a ground-based survey with $\sim 1^{\prime \prime}$ seeing is likely to miss $\sim 60 \%$ of these lenses. There is also a tail of lenses with separations $\Delta \theta>5^{\prime \prime}$. These large-separation lenses, and the observational difficulties in identifying them, have been previously explored both analytically (Narayan \& White 1988; Kochanek 1995) and with numerical simulations (Cen et al. 1994; Wambsganss et al. 1995).

The magnification bias is determined by the distribution of image magnifications and by the source luminosity function. Denoting the probability distribution of magnifications by $q(A)$ (independent of $z_{L}$ and $z_{S}$ for the SIS), and the number counts of sources per unit flux at a flux $F$ by

TABLE 1

FRACTION OF LENSED SOURCES

\begin{tabular}{ccccc}
\hline \hline & \multicolumn{3}{c}{ PS HaLOS } & GaLAXIES \\
\cline { 2 - 5 } CosmoloGICAL & $\begin{array}{c}z_{S}=10 \\
\text { Model }\end{array}$ & $\begin{array}{c}z_{S}=5 \\
(\%)\end{array}$ & $\begin{array}{c}z_{S}=2 \\
(\%)\end{array}$ & $\begin{array}{c}z_{S}=2 \\
(\%)\end{array}$ \\
\hline$\Lambda$ CDM $\ldots \ldots \ldots$ & 5.5 & 3.3 & 1.0 & 0.80 \\
OCDM $\ldots \ldots \ldots$ & 4.5 & 2.5 & 0.70 & 0.38 \\
SCDM $\ldots \ldots \ldots$ & 5.5 & 3.4 & 1.3 & 0.26 \\
\hline
\end{tabular}

NoTE.- In all cases we have assumed a factor of 5 from magnification bias. 


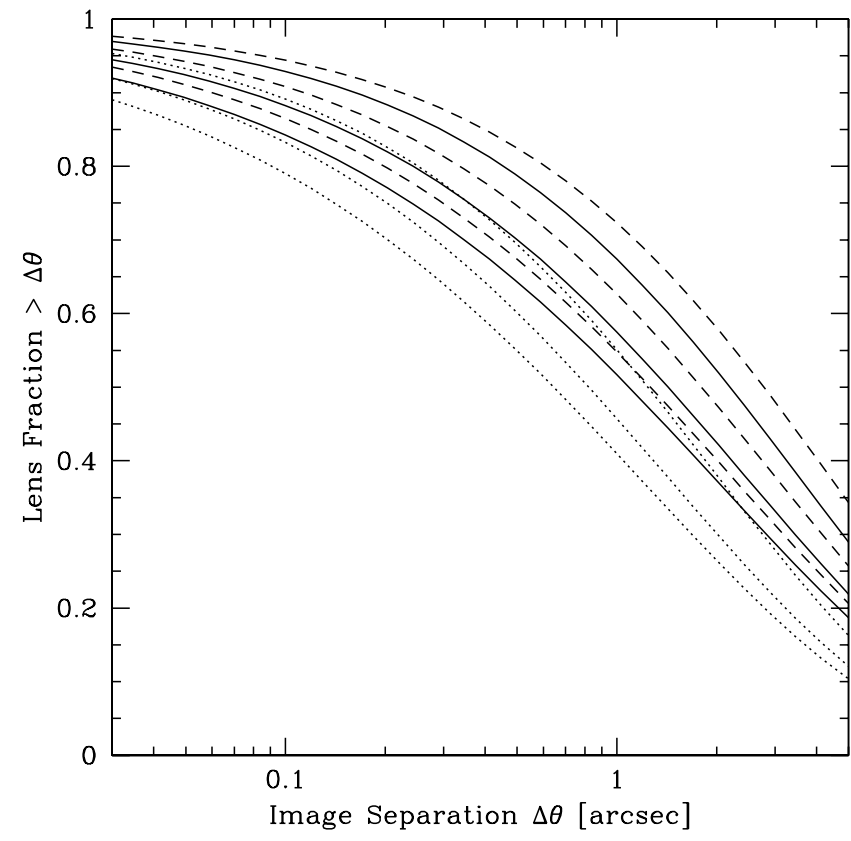

FIG. 3.-Integral distribution of image separation for PS halos in $\Lambda C D M$ (solid curves), OCDM (dashed curves), and SCDM (dotted curves). The results in each cosmological model are shown for sources at $z_{S}=2,5$, and 10 , from top to bottom.

$d N / d F$, the fraction of lensed sources at the observed flux $F$ is increased by a bias factor

$$
B=\left.\int \frac{d N}{d F}\right|_{F / A}\left(\left.\frac{d N}{d F}\right|_{F}\right)^{-1} q(A) \frac{d A}{A} .
$$

As noted above, NGST will resolve almost all double images, so we count them as two apparent sources. Thus, we compute the bias factors separately for the two images, using $q(A)=2 /(A-1)^{3}$ and $A>2$ for the brighter image, and $q(A)=2 /(A+1)^{3}$ and $A>0$ for the fainter image. We then find the sum, which is dominated by the brighter image of the two. This sum includes the contributions to sources observed at a flux $F$ from all lensed images (each of which is either the bright image or the faint image of a lensed pair). The product of the resulting bias factor and the lensing optical depth yields the fraction of all apparent sources that are part of a lensed system. We note that any attempt to estimate the magnification bias of high-redshift sources is highly uncertain at present due to several tentative assumptions about their characteristic mass-to-light ratio, star formation history, initial stellar mass function, dust-extinction amplitude, and quasar formation history.

Figure 4 illustrates the magnification bias for the NGST number-count model of Haiman \& Loeb (1999; 1997), who assumed cosmological parameters nearly equivalent to our $\Lambda \mathrm{CDM}$ model. Solid lines show mini-quasars, dashed lines show galaxies undergoing starbursts that convert $20 \%$ of the gas of each halo into stars, and dotted lines show starbursts that use only $2 \%$ of the gas of each halo. For each type of source, we show separate curves corresponding to all sources at redshifts $z_{S}>5$ or to all sources at redshifts $z_{S}>10$. Although the $z_{S}>10$ number counts are smaller, they are steeper than the $z_{S}>5$ counts and produce a larger magnification bias. Similarly, for low (2\%) star-formation efficiency, galaxies are detected only if they lie in relatively massive halos, which have a steeper mass function and thus

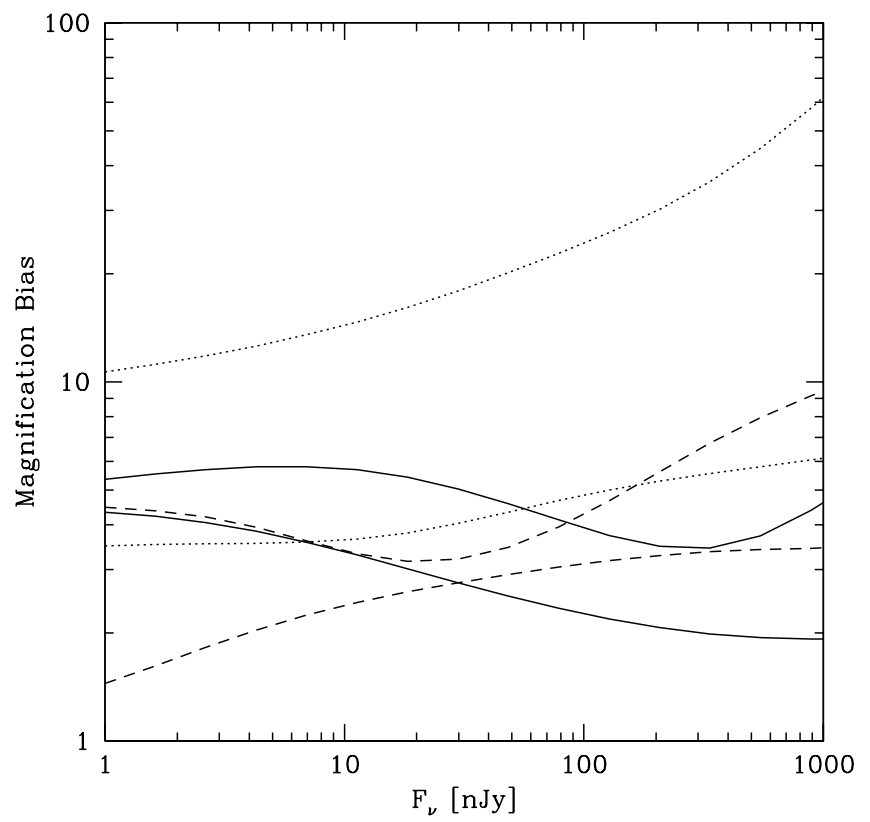

Fig. 4.-Magnification bias for the Haiman \& Loeb (1999) number counts in $\Lambda \mathrm{CDM}$ (extrapolated as power laws to fluxes fainter than 0.1 $\mathrm{nJy})$. Solid lines show mini-quasars, dashed lines show galaxies undergoing starbursts, which convert $20 \%$ of the gas in each halo into stars, and dotted lines show starbursts that use only $2 \%$ of the gas in each halo. For each type of source, the lower curve is for all sources at $z_{S}>5$ and the higher one is for all sources at $z_{S}>10$.

a larger magnification bias than for a higher star-formation efficiency. These results indicate a magnification bias around 3-6, but this factor could be much higher if the actual number counts are only somewhat steeper than predicted by these models. Indeed, the number counts fall off roughly as power laws, $d N / d F_{v} \propto F_{v}^{-\beta}$ with $\beta \sim 2-2.5$, while for the SIS, the magnification bias diverges at the critical value $\beta=3$. Using Figure 4, Table 1 , and the number counts of Haiman \& Loeb (1999), the estimated number of sources (or lensed sources, values given in parentheses) above $1 \mathrm{nJy}$ per $4^{\prime} \times 4^{\prime}$ field of view is 90 (5) for $z>10$ quasars, 300 (12) for $z>5$ quasars, 400 (17) for $z>10$ galaxies with $20 \%$ star-formation efficiency, $10^{4}$ (200) for $z>5$ galaxies with $20 \%$ efficiency, 20 (1) for $z>10$ galaxies with $2 \%$ efficiency, and $2 \times 10^{3}$ (30) for $z>5$ galaxies with $2 \%$ efficiency. Note, however, that the number counts for galaxies are reduced when we include the fact that most galaxies are resolved by NGST and cannot be treated as point sources (see $\S 3$ ).

We have assumed that each lensing halo can be approximated as an SIS, although the mass distributions in actual halos might be more complicated. Numerical simulations of pure dark matter indicate a roughly universal profile (NFW) with a $1 / r$ density profile in the core. This result is supported by very high resolution simulations of a small number of halos (Moore et al. 1999), although simulations of large numbers of halos typically find a shallower inner density profile, in agreement with observed rotation curves of dark-matter-dominated galaxies (Kravtsov et al. 1998). In addition, galaxy halos undergo adiabatic compression when the baryons cool and contract (e.g., Flores et al. 1993). Halos with the NFW profile have a smaller lensing cross section than the SIS, but this is partly compensated for by the higher mean magnification and thus the higher magnifi- 
cation bias produced by NFW lenses (C. R. Keeton, in preparation). In the above discussion, we have also assumed spherical symmetry. If the SIS is made ellipsoidal, with an ellipticity of 0.3 , then the total lensing cross section is changed only slightly, but lenses above a total magnification of $\sim 8$ are then mostly four-image systems (see, e.g., Kochanek 1996b). We have also assumed that each halo acts as an isolated lens, while in reality galaxies are clustered and many galaxies lie in small groups. The large dark matter halo associated with the group may combine with the halos of the individual galaxies and enhance their lensing cross section. External shear due to group halos will also tend to increase the fraction of four-image systems. On the other hand, dust extinction may reduce the number of lensed systems below our estimates, especially since highredshift galaxies are observed at rest-frame UV wavelengths. Significant extinction may arise from dust in the source galaxy itself as well as dust in the lens galaxy, if the image path passes sufficiently close to the center of the lens galaxy.

\section{SIZE DISTRIBUTION OF HIGH-REDSHIFT DISK GALAXIES}

\subsection{Semianalytic Model}

The formation of disk galaxies within hierarchical models of structure formation was explored by Fall \& Efstathiou (1980). More recently, the distribution of disk sizes was derived and compared to observations by DSS and MMW. In order to estimate the ability of NGST to resolve highredshift disks, we adopt the simple model of an exponential disk in an SIS halo. We consider a halo of mass $M$, virial radius $r_{\text {vir }}$, total energy $E$, and angular momentum $J$, for which the spin parameter is defined as

$$
\lambda \equiv J|E|^{1 / 2} G^{-1} M^{-5 / 2} .
$$

If the disk mass is a fraction $m_{d}$ of the halo mass and its angular momentum is a fraction $j_{d}$ of that of the halo, then the exponential scale radius of the disk is given by (MMW)

$$
R_{d}=\frac{1}{\sqrt{2}}\left(\frac{j_{d}}{m_{d}}\right) \lambda r_{\mathrm{vir}}
$$

The observed distribution of disk sizes suggests that the specific angular momentum of the disk is similar to that of the halo (see DSS and MMW), and so we assume $j_{d} / m_{d}=1$. The distribution of disk sizes is then determined ${ }^{2}$ by the PS halo abundance and by the distribution of spin parameters. The latter approximately follows a lognormal distribution,

$$
p(\lambda) d \lambda=\frac{1}{\sigma_{\lambda} \sqrt{2 \pi}} \exp \left[-\frac{\ln ^{2}(\lambda / \bar{\lambda})}{2 \sigma_{\lambda}^{2}}\right] \frac{d \lambda}{\lambda},
$$

where $\bar{\lambda}=0.05$ and $\sigma_{\lambda}=0.5$ following MMW, who determined these values based on the $N$-body simulations of Warren et al. (1992). Unlike MMW, we do not include a lower cutoff on $\lambda$ due to disk instability. If a dense bulge exists, it can prevent bar instabilities, or if a bar forms it may be weakened or destroyed when a bulge subsequently forms (Sellwood \& Moore 1999).

\footnotetext{
${ }^{2}$ For a halo of a given mass and redshift, we determine $r_{\text {vir }}$ using NFW and equation (6) of Bryan \& Norman (1998); see also § 2.1.
}

The distribution of disks is truncated at the low-mass end due to the fact that gas pressure inhibits baryon collapse and disk formation in shallow potential wells, i.e., in halos with a low circular velocity $V_{c}$. In particular, photoionization heating by the cosmic UV background heats the intergalactic gas to a characteristic temperature of $\sim 10^{4} \mathrm{~K}$ and prevents it from settling into systems with a lower virial temperature. Using a spherical collapse code, Thoul \& Weinberg (1996) found a reduction of $\sim 50 \%$ in the collapsed gas mass due to heating for a halo of $V_{c}=50 \mathrm{~km} \mathrm{~s}^{-1}$ at $z=2$, and a complete suppression of infall below $V_{c}=30$ $\mathrm{km} \mathrm{s}^{-1}$. Three-dimensional numerical simulations (Quinn, Katz, \& Efstathiou 1996; Weinberg, Hernquist, \& Katz 1997; Navarro \& Steinmetz 1997) found a suppression of gas infall into even larger halos with $V_{c} \sim 75 \mathrm{~km} \mathrm{~s}^{-1}$. We adopt a typical cutoff value of $V_{\text {cut }}=50 \mathrm{~km} \mathrm{~s}^{-1}$ in the PS halo function, requiring $V_{c}>V_{\text {cut }}$ for the formation of disks. We note, however, that the appropriate $V_{\text {cut }}$ could be lower at both very low and very high redshifts, when the cosmic UV background was weak. In particular, the decline of the UV background at $z \sim 1$ allowed gas to condense in halos down to $V_{c} \sim 25 \mathrm{~km} \mathrm{~s}^{-1}$ (Kepner, Babul, \& Spergel 1997). Similarly, gaseous halos that had formed prior to reionization, when the cosmic UV background was negligible, could have survived photoionization heating at later times as long as they satisfied $V_{c} \gtrsim 13 \mathrm{~km} \mathrm{~s}^{-1}$ (Barkana \& Loeb 1999).

Aside from its relevance to lensing studies, the distribution of disk sizes is useful for assessing the level of overlap of sources on the sky, namely, the confusion noise. We first compute the geometric optical depth of galactic disks, i.e., the fraction of the sky covered by galactic disks. This corresponds to the probability of encountering a galactic disk (within one exponential scale length) inside an infinitesimal aperture. Averaging over all random orientations, a circular disk of radius $R_{d}$ at redshift $z_{S}$ occupies an angular area of $2\left(R_{d} / D_{O S}\right)^{2}$. The total optical depth then depends on $V_{\text {cut }}$. For $\Lambda \mathrm{CDM}$ with $V_{\text {cut }}=50 \mathrm{~km} \mathrm{~s}^{-1}$, we find the geometric optical depth to be $2.0 \times 10^{-4}$ when integrated over all $z>10$ sources, $5.5 \times 10^{-3}$ for $z>5$ sources, $1.7 \%$ for $z>3$ sources, $4.6 \%$ for $z>1$ sources, and $6.8 \%$ for sources at all redshifts. If we lower $V_{\text {cut }}$ to $30 \mathrm{~km} \mathrm{~s}^{-1}$, the optical depth becomes $8.8 \times 10^{-4}$ for $z>10$ sources, $3.5 \%$ for $z>3$ sources, and $11.3 \%$ for all source redshifts.

A more realistic estimate of confusion noise must include the finite resolution of the instrument as well as its detection limit for faint sources. We characterize the instrument's resolution by a minimum circular aperture of angular diameter $\theta_{a}$. We include as sources only those galactic disks that are brighter than some threshold. This threshold is dictated by $F_{v}^{\mathrm{ps}}$, the minimum spectral flux ${ }^{3}$ required to detect a pointlike source (i.e., a source that is much smaller than $\theta_{a}$ ). For an extended source of diameter $\theta_{s} \gg \theta_{a}$, we assume that the signal-to-noise ratio can be improved by using a larger aperture, with diameter $\theta_{s}$. The noise amplitude scales as the square root of the number of noise (sky) photons, or the square root of the corresponding sky area. Thus, the total flux needed for detection of an extended source at a given signal-to-noise ratio threshold is larger than $F_{v}^{\text {ps }}$ by a factor of $\theta_{s} / \theta_{a}$. We adopt a simple interpolation formula between the regimes of pointlike and extended sources,

\footnotetext{
${ }^{3}$ Note that $F_{v}^{\text {ps }}$ is the total spectral flux of the source, not just the portion contained within the aperture.
} 
and assume that a source is detectable if its flux is at least $\left[1+\left(\theta_{s} / \theta_{a}\right)^{2}\right]^{1 / 2} F_{v}^{\mathrm{ps}}$.

We can now compute the "intersection probability," namely, the probability of encountering a galactic disk (within one exponential scale length) anywhere inside the aperture of diameter $\theta_{a}$. A face-on circular disk of diameter $\theta_{s}=2 R_{d} / D_{O S}$ will overlap the aperture if its center lies within a radius of $\left(\theta_{a}+\theta_{s}\right) / 2$ about the center of the aperture. Assuming a random orientation of the disk, the average cross section is then $\pi \theta_{a}^{2} / 4+1.323 \theta_{a} \theta_{s}+\theta_{s}^{2} / 2$. We integrate this cross section over the spin parameter distribution and over the abundance of halos at all masses and redshifts. The resulting intersection probability is closely related to the confusion noise. If this probability is small, then individual sources are resolved from each other, since the aperture typically contains at most a single detectable source. We can also obtain a limit on the confusion noise from sources below the flux detection threshold by computing the same intersection probability but including sources at all fluxes.

The flux, $F_{v}$, of a given disk depends on its mass-to-light ratio, which in turn depends on its star formation history and stellar mass function. We adopt a semianalytic starburst model similar to that of Haiman \& Loeb (1999), but different in detail. We assume that each halo of mass $M$ hosts a disk of mass $m_{d} M$, of which a fraction $f_{d}$ participates in star formation. Adopting a cosmological baryon density of $\Omega_{b} h^{2}=0.02$, we define the star formation efficiency $\eta$ so that $f_{d} m_{d}=\eta\left(\Omega_{b} / \Omega_{0}\right)$. We assume a fixed universal value of $\eta$, and illustrate our results for a high efficiency of $\eta=20 \%$ (assumed unless indicated otherwise) and for a low efficiency of $\eta=2 \%$. These values cover the range of efficiencies suggested by observations of the metallicity of the Ly $\alpha$ forest at $z=3$ (Haiman \& Loeb 1999) and the cumulative mass density of stars in the universe at present (Fukugita, Hogan, \& Peebles 1998). Note that $\eta=20 \%$ and a particular value of $F_{v}^{\mathrm{ps}}$ are equivalent to $\eta=2 \%$ and a tenfold decrease in $F_{v}^{\mathrm{ps}}$.

In order to determine the mass-to-light ratio of a halo of mass $M$ at a redshift $z$, we assume that the mass $\eta\left(\Omega_{b} / \Omega_{0}\right) M$ is distributed in stars with a Salpeter mass function $\left(d N \propto m^{-\alpha} d m\right.$ with $\left.\alpha=2.35\right)$ from $1 M_{\odot}$ up to $100 M_{\odot}$. If the mass function were extended to masses below $1 M_{\odot}$, the additional stars would contribute significant mass but little luminosity, so this would essentially be equivalent to a reduction in $\eta$. We use the stellar population code of Sternberg (1998) with $Z=0.001$ stellar tracks and $Z=0.006$ stellar spectra. We assume that the age of the stellar population equals that of the dark matter halo, whose age is determined from its merger history. The formation redshift, $z_{\text {form }}>z$, is defined as the time at which half the mass of the halo was first contained in progenitors more massive than a fraction $f$ of $M$. We set $f=0.5$ and estimate the formation redshift (and thus the age) using the extended PS formalism (see, e.g., Lacey \& Cole 1993). At high redshifts, the young age of the universe and high halo merger rate imply young stellar populations that are especially bright at rest-frame UV wavelengths. At each redshift $z$, we calculate the halo spectral flux by averaging the composite stellar spectrum over the wavelengths corresponding to the observed NGST spectral range of $0.6-3.5 \mu \mathrm{m}$. We also include a Ly $\alpha$ cutoff in the spectrum due to absorption by the dense Ly $\alpha$ forest at all redshifts up to that of the source. We do not, however, include dust extinction. Despite the generally low metal- licity at high redshifts, extinction could be significant, since observations correspond to rest-frame UV wavelengths (Loeb \& Haiman 1997).

Our starburst model is expected to describe galaxies at high redshifts, but it may fail at redshifts $z \lesssim 2$. The model relies on two key assumptions, namely, that stars form in disks, and that the stars in each galaxy have formed since the last major merger of its halo. At high redshifts, the fraction of gas that has collapsed into halos is small, and the fraction that has turned into stars is even smaller. Thus, a high-redshift galaxy is expected to be gas-rich whether it forms in a merger or accretes most of its gas from the intergalactic medium. Such a galaxy is likely to form most of its stars in a disk after the gas cools and settles onto a plane. At low redshifts, on the other hand, disk galaxies may have converted most of their gas into stars by the time they merge. In this case, the merger may form a massive elliptical galaxy rather than a disk-dominated galaxy. Indeed, elaborate semianalytic models indicate that the stars in elliptical galaxies are typically much older than their halo merger age (e.g., Thomas \& Kauffmann 1999), in agreement with the red colors of ellipticals, which suggest old stellar populations. Although the increased presence of elliptical galaxies invalidates our model for the mass-to-light ratios of galaxies at low redshifts, our results for the size distribution of galaxies may remain approximately valid. Theoretical considerations based on the virial theorem, as well as numerical simulations, suggest that the characteristic size of a galactic merger remnant is smaller by a factor of $\lesssim 1.5$ than the size expected for a disk galaxy of the same mass and velocity dispersion (Hausman \& Ostriker 1978; Hernquist, Spergel, \& Heyl 1993).

\subsection{Numerical Results}

Figure 5 shows the total intersection probability as a function of limiting flux (right panel) for all sources with $z>0,2,5$, and 10, from top to bottom. The total probability is dominated by the contribution of sources at low redshifts, which is relatively insensitive to the limiting flux (or to $\eta$ ). All curves assume the $\Lambda$ CDM model with a circularvelocity cutoff for the host halo of $V_{\text {cut }}=50 \mathrm{~km} \mathrm{~s}^{-1}$. The aperture diameter is chosen to be $\theta_{a}=0.06$, close to the expected $N G S T$ resolution at $2 \mu \mathrm{m}$. With $F_{v}^{\mathrm{ps}}=1 \mathrm{nJy}$, the total intersection probability for all redshifts is $8.9 \%$ (or $5.6 \%$ if $\eta=2 \%$ ) in $\Lambda \mathrm{CDM}$ (it is also $10 \%$ or less in the SCDM and OCDM models). The probability increases to $15 \%(6.2 \%$ if $\eta=2 \%)$ if $V_{\text {cut }}=30 \mathrm{~km} \mathrm{~s}^{-1}$ instead of $50 \mathrm{~km}$ $\mathrm{s}^{-1}$. The contribution from sources at $z>5$ is $1.0 \%$ $\left(9.0 \times 10^{-4}\right.$ if $\left.\eta=2 \%\right)$. Thus, the chance for overlapping sources will be small for NGST. If the resolution were $\theta_{a}=$ 0 . 12 , the probability would be $12 \%(6.6 \%$ if $\eta=2 \%)$ for all redshifts and $1.7 \%(0.14 \%$ if $\eta=2 \%)$ for sources at $z>5$. If we include all sources regardless of flux, then the probability becomes independent of $\eta$, and (with $\theta_{a}=0$.06) it equals $9.1 \%$ if $V_{\text {cut }}=50 \mathrm{~km} \mathrm{~s}^{-1}$ and $18.8 \%$ if $V_{\text {cut }}=30 \mathrm{~km} \mathrm{~s}^{-1}$. The contribution from sources below the detection threshold is small due to the $V_{c}$ cutoff, i.e., the fact that the photoionizing background prevents the formation of galaxies in small dark matter halos. This fact should eventually result in a turnover, where the number counts do not increase with decreasing flux. However, the turnover occurs somewhat below $1 \mathrm{nJy}$, a flux that is much smaller than the detection threshold of current observations such as the Hubble Deep Field. 


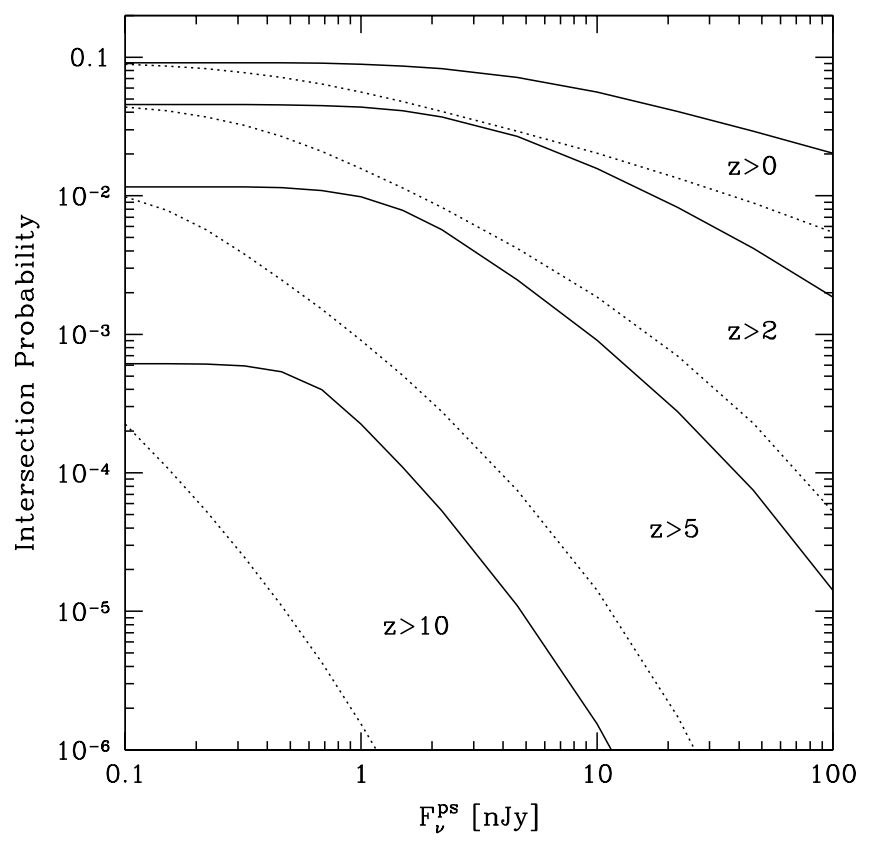

FIG. 5.-Probability of encountering a galactic disk inside the NGST aperture of diameter 0.06 . We show this intersection probability as a function of limiting point-source flux for a high-efficiency $(\eta=20 \%$, solid curves) and a low-efficiency ( $\eta=2 \%$, dotted curves) of star formation. In each case, the curves include all disks with $z>0,2,5$, and 10, respectively, from top to bottom. We assume the $\Lambda \mathrm{CDM}$ model and a circular-velocity cutoff for the host halo of $V_{\text {cut }}=50 \mathrm{~km} \mathrm{~s}^{-1}$.

In summary, we have shown that confusion noise for NGST will be low, assuming that there is one galaxy per halo and that the luminous stars form primarily in disks. Note that we have not included the possible confusion noise from multiple galaxies per halo, from clustered or interacting galaxies, or from galaxies being observed as separate fragments rather than smooth disks. We also have not included the confusion noise from stars and other sources in our own galaxy. Also note that with no flux limit on sources, the intersection probability approaches unity only if the aperture is increased to 0 .". .

Our model predicts the size distribution of galaxies at various redshifts. Figure 6 shows the fraction of the total number counts contributed by sources with diameters greater than $\theta$, as a function of $\theta$. The size distributions are shown for a high efficiency $(\eta=20 \%$; Fig. 6 , solid curves $)$ and a low efficiency $(\eta=2 \%$, dotted curves) of star formation. Each curve is marked by the lower limit of the corresponding redshift range, with " 0 " indicating sources with $0<z<2$, and similarly for $2<z<5,5<z<10$, and $z>10$. All curves include a cutoff of $V_{\text {cut }}=50 \mathrm{~km} \mathrm{~s}^{-1}$ and a limiting point-source flux of $1 \mathrm{nJy}$, and all are for the $\Lambda \mathrm{CDM}$ model. The vertical dashed line in Figure 6 indicates the NGST resolution of 0 ".06. Note that increasing $\eta$ leads to a decrease in the typical angular size of galaxies, since the set of observable galaxies then includes galaxies that are less massive, and thus generally smaller. However, a tenfold increase in $\eta$ decreases the observed angular sizes of $z>10$ galaxies by only a factor of 2 .

The typical observed size of faint disks (i.e., of all disks down to $1 \mathrm{nJy})$ is 0.4 for sources at $0<z<2,0$ ". 2 for sources at $2<z<5,0$ ". 10 (or 0 ". 15 if $\eta=2 \%$ ) for sources at $5<z<10$, and 0.065 (or 0 " 11 if $\eta=2 \%$ ) for sources at $z>10$. Roughly $60 \%$ of all $z>10$ sources (or $90 \%$ if

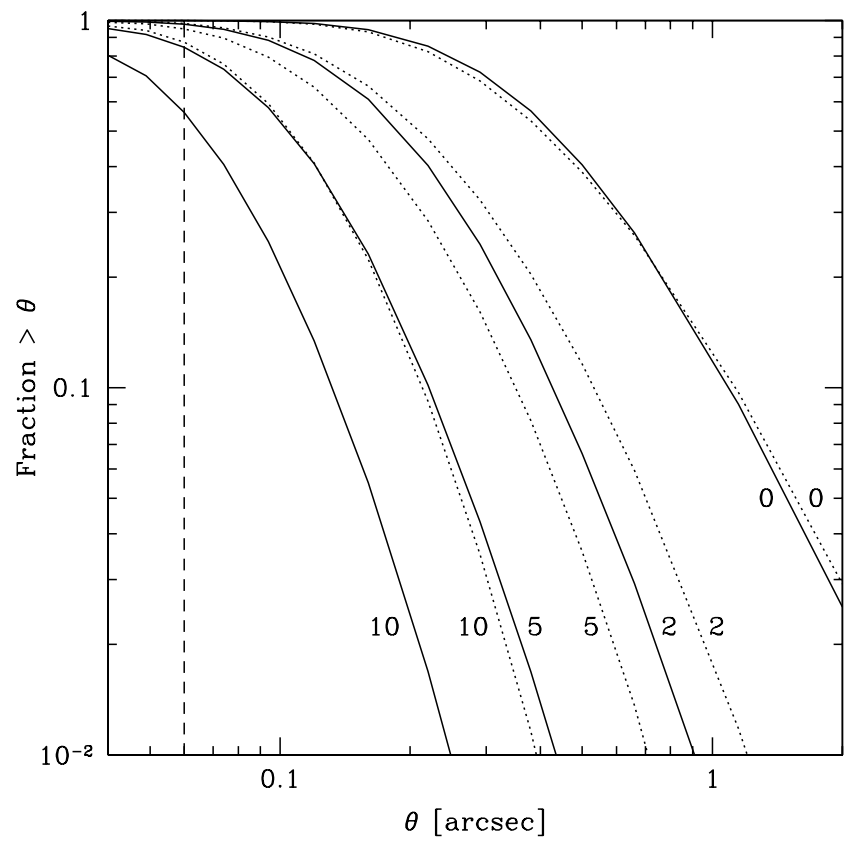

FIG. 6.-Distribution of galactic disk sizes in various redshift intervals, in the $\Lambda$ CDM model. Given $\theta$ in arcseconds, each curve shows the fraction of the total number counts contributed by sources larger than $\theta$. The diameter $\theta$ is measured out to one exponential scale length. We assume either a high efficiency $(\eta=20 \%$, solid curves) or a low efficiency $(\eta=2 \%$, dotted curves) of star formation. We indicate next to each curve the lower limit of the redshift interval. Thus, " 0 " indicates sources with $0<z<2$, and similarly we consider sources with $2<z<5,5<z<10$, and $z>10$. All curves include a cutoff velocity of $V_{\text {cut }}=50 \mathrm{~km} \mathrm{~s}^{-1}$ and a limiting point source flux of $1 \mathrm{nJy}$. The vertical dashed line indicates the NGST resolution of 0.06 .

$\eta=2 \%$ can be resolved by NGST, and the fraction is at least $85 \%$ among lower redshift sources. Thus, the high resolution of NGST should make most of the detected sources useful for weak lensing. If reliable shape measurements require a diameter equal to twice the resolution scale (probably overly pessimistic), then the useful $(\theta>0$ ".12) fractions are $13 \%$ for $z>10,40 \%$ for $5<z<10$, and $80 \%$ for $2<z<5$ sources. If $\eta=2 \%$, the corresponding fractions are $40 \%$ for $z>10,65 \%$ for $5<z<10$, and $80 \%$ for $2<z<5$. These results are all in the $\Lambda$ CDM model, but disk sizes in the SCDM and OCDM models differ by only about $10 \%$.

As noted by Schneider \& Kneib (1998), ground-based telescopes that are not equipped with adaptive optics or interferometry would be unable to resolve most of the highredshift sources, even if they could reach the same flux sensitivity as NGST. For example, a ground-based survey down to $1 \mathrm{nJy}$ with, e.g., $0^{\prime \prime} .75$ seeing at $2 \mu \mathrm{m}$, could resolve only $0.003 \%$ of the $z>10$ sources, with corresponding fractions of $0.1 \%$ for $5<z<10$ and $2 \%$ for $2<z<5$. If $\eta=2 \%$, then the resolved fractions are $0.03 \%$ for $z>10,0.8 \%$ for $5<z<10$, and $4 \%$ for $2<z<5$. Thus, the high resolution of NGST is crucial for resolving faint galaxies at the redshifts of interest.

Current observations of galaxy sizes at $z>2$ are inadequate for a detailed comparison with our models. J. P. Gardner \& S. Satyapal (in preparation) have determined the sizes of galaxies in the Hubble Deep Field South, finding typical half-light radii of 0.1 with a very large scatter. This 
sample likely includes a wide range of redshifts, and it is expected to be strongly biased toward small galaxy sizes. Given the steep luminosity function of the detected galaxies, most of them are detected very close to the detection limit, especially those at high redshift. Of course, galaxies near the flux threshold can be detected only if they are nearly point sources, while large galaxies are excluded from the sample because of their low surface brightness.

Since most galaxies will be resolved by NGST, predictions for the total number counts are affected by the higher flux needed for the detection of extended objects relative to point sources. For a point-source flux limit of $1 \mathrm{nJy}$ and $\eta=20 \%$, the total number counts are reduced (relative to a size-independent flux limit of $1 \mathrm{nJy}$ ) by a factor of 2 for $z>10$ and by only $10 \%$ for $5<z<10$. The reduction for $z<10$ sources is small if $\eta=20 \%$, since in this case the total flux of most $z<10$ sources is greater than $1 \mathrm{nJy}$, and these galaxies can still be detected even as extended objects. However, the reduction in number counts is more significant if $\eta=2 \%$, with a factor of 8 for $z>10,4$ for $5<z<10$, and 2.5 for $2<z<5$.

We show in Figure 7 the resulting prediction for the redshift distribution of the galaxy population observed with $N G S T$. We assume a $\Lambda$ CDM model and plot $d N / d z$, where $N$ is the number of galaxies per NGST field of view. The solid curve assumes a high efficiency $(\eta=20 \%)$ of star formation, and the dashed curve assumes a low efficiency $(\eta=2 \%)$. All curves assume a limiting point-source flux of 1 $\mathrm{nJy}$. The total number per field of view of galaxies at all redshifts is $N=59,000$ for $\eta=20 \%$ and $N=15,000$ for $\eta=2 \%$. The fraction of galaxies above $z=5$ is sensitive to the value of $\eta$ (it is $40 \%$ for $\eta=20 \%$ and $7.4 \%$ for $\eta=2 \%$ ), but the number of $z>5$ galaxies is large $(\sim 1000)$ even for the low efficiency. The number of $z>5$ galaxies predicted in

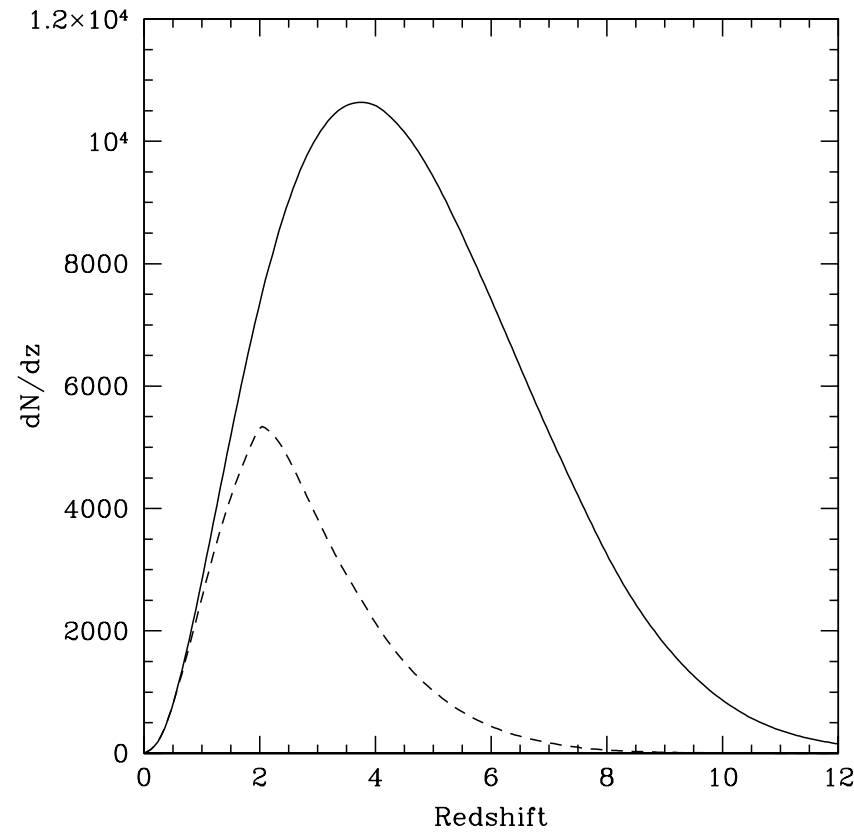

FIG. 7.-Predicted redshift distribution of galaxies observed with $N G S T$. The distribution in the $\Lambda$ CDM model is shown for a high efficiency ( $\eta=20 \%$, solid curve) or a low efficiency $(\eta=2 \%$, dashed curve) of star formation. The plotted quantity is $d N / d z$, where $N$ is the number of galaxies per NGST field of view. All curves assume a limiting point-source flux of $1 \mathrm{nJy}$.
SCDM is close to that in $\Lambda \mathrm{CDM}$, but in OCDM there are twice as many $z>5$ galaxies.

\subsection{The Surface Brightness of Lensed Sources}

In our estimates of the lensing rate in $\S 2$, we implicitly made two important assumptions: (1) that the source is smaller than the image separation, so that the two images of the source are not blended; and (2) that the surface brightness of the background source is comparable to or higher than that of the foreground lens; otherwise, the background source could not be detected when it is superimposed on the lens galaxy. These assumptions are trivially justified for the pointlike images of quasars. In the context of galactic sources, we can apply our estimates of disk sizes to test these two assumptions quantitatively.

A lensed galaxy is generally much smaller than the separation of its two images. The combination of Figures 3 and 6 shows that, regardless of the source redshift, the typical image separation is at least 4 times as large as the typical diameter of a source galaxy detected by NGST. Thus, the majority of all lensed sources will not be blended. Note, however, that if ellipticity or shear are included, then some of the resulting four-image systems may include arcs produced by several blended images.

In order to compare the surface brightness of source galaxies to that of lens galaxies, we calculate the redshift evolution of the mean surface brightness of galaxies. At high redshifts, we can apply our disk starburst model to find the surface brightness of a galaxy from the predicted size, mass, and mass-to-light ratio of its disk. We compute the average surface brightness (as observed in the NGST spectral range) out to one exponential scale length. Figure 8 shows this surface brightness, $\mu$ (expressed in $\mathrm{nJy} \operatorname{arcsec}^{-2}$ ), averaged over all galaxies at each redshift in the $\Lambda \mathrm{CDM}$ model only (since the OCDM and SCDM models yield very similar results). Solid lines show the mean at $z>2$, where galaxies are weighed by their number density and their mass-to-light ratios are derived from the starburst model. As discussed at the end of $\S 3.1$, although our model for the size distribution of galaxies should remain approximately valid at low redshifts, the starburst model may fail to predict the correct mass-to-light ratio of the stellar population at $z \lesssim 2$, particularly for the lens galaxies. These lenses tend to be massive elliptical galaxies, with stellar populations that may be much older than the merger timescale assumed in our starburst model. In order to estimate the surface brightness of lens galaxies, we adopt a simple alternative model in which all their stars are uniformly old. The dashed lines in Figure 8 show (for $z<2$ ) the mean surface brightness of lensing galaxies (i.e., where galaxies are weighed by the product of their number density and their lensing cross section), assuming that their stars formed at $z=5$. In each case (i.e., for source galaxies or for lens galaxies), the upper curve assumes a high efficiency $(\eta=20 \%)$ and the lower curve assumes a low efficiency $(\eta=2 \%)$ of incorporating baryons into stars in the associated halos. All curves include a cutoff velocity of $V_{\text {cut }}=50 \mathrm{~km} \mathrm{~s}^{-1}$ and a limiting point-source flux of $1 \mathrm{nJy}$.

As is apparent from Figure 8, the mean surface brightness of galaxies varies, for a fixed $\eta$, by a factor of $\lesssim 2$ over all redshifts above 2 , despite the large range in luminosity distances from the observer. Several different factors combine to keep the surface brightness nearly constant. Except for redshift factors, the surface brightness is proportional to the 


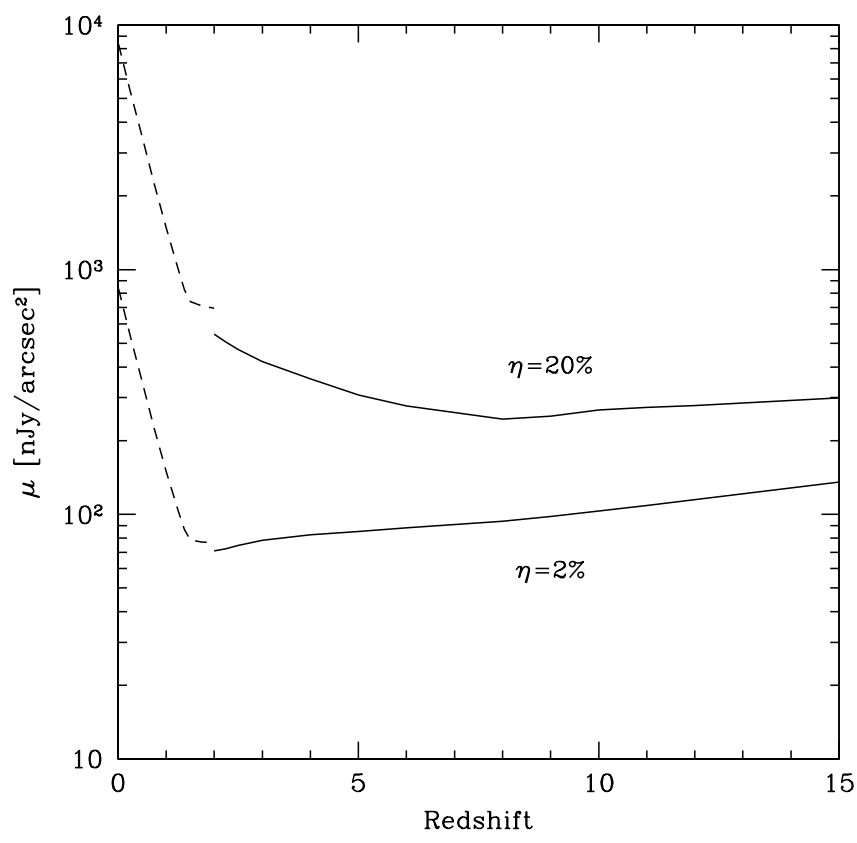

FIG. 8.-Redshift evolution of the mean surface brightness $\mu$ of galaxies, in the $\Lambda \mathrm{CDM}$ model, averaged over the NGST wavelength band. In calculating this mean, the contribution of each disk is its average surface brightness out to one exponential scale length. Solid lines show (for $z>2$ ) the mean calculated for all galaxies weighed by their number density, with the mass-to-light ratios derived from the starburst model. Dashed lines show (for $z<2$ ) the mean for lens galaxies (i.e., weighed by the product of their number density and their lensing cross section), with the mass-to-light ratios derived from assuming that all their stars formed at $z=5$. In each case, the upper curve assumes a high efficiency $(\eta=20 \%)$ and the lower curve assumes a low efficiency $(\eta=2 \%)$ of converting gas into stars. All curves include a cutoff velocity of $V_{\text {cut }}=50 \mathrm{~km} \mathrm{~s}^{-1}$ and a limiting pointsource flux of $1 \mathrm{nJy}$.

luminosity over the square of the disk radius, and the luminosity is in turn equal to the disk mass divided by its massto-light ratio. Although the typical mass of halos decreases at high redshifts, two other effects tend to increase the surface brightness. First, high-redshift disks are compact due to the increased mean density of the universe. The second effect results from the low mass-to-light ratio of the young stellar populations of high-redshift disks, which makes these galaxies highly luminous despite their small masses. For example, the mean ratio of halo mass to disk luminosity for $z=2$ galaxies (with $\eta=20 \%$ and $F_{v}^{\text {ps }}=1$ $\mathrm{nJy}$ ) is 14 in solar units, and this decreases to 3.8 at $z=5$ and 1.2 at $z=10$. This evolution in the mass-to-light ratio includes the so-called $K$-correction, i.e., the fact that for higher redshift sources, the NGST filter corresponds to shorter rest-frame wavelengths.

Acting alone, the factors discussed above would result in a sharp increase with redshift in the surface brightness of galaxies. Additional redshift effects, however, counterbalance these other factors. According to the Tolman surface brightness law, the expansion of the universe yields a factor of $(1+z)^{-4}$ regardless of the values of the cosmological parameters. This redshift factor dominates and produces an overall decrease in $\mu$ among lens galaxies at low redshifts (up to $z \sim 1.5$ ). At these low redshifts, all galaxies are detected regardless of $\eta$, so the overall $\mu$ is exactly proportional to $\eta$. At higher redshifts, the $1 \mathrm{nJy}$ flux limit preferentially removes low surface brightness galaxies from the detected sample. The resulting bias toward high surface brightness is larger if $\eta=2 \%$, and this decreases the difference in $\mu$ between the cases of $\eta=2 \%$ and $\eta=20 \%$. The mass-to-light ratio begins to decrease rapidly at $z \gtrsim 1.5$, and at $z>2$ the various factors combine to produce a slow variation in $\mu$.

Although there is only a modest redshift evolution in the surface brightness of galaxies, there is an additional difficulty in detecting lensed sources. Lensing galaxies are biased toward larger circular velocities, i.e., toward larger masses at each redshift. Since a galaxy that is more massive is usually also more luminous, its surface brightness tends to be larger. As shown in Figure 8, this tendency makes the mean surface brightness of lenses somewhat higher than that of sources, despite our assumption of an old stellar population in lens galaxies. Consider, for example, a source at redshift 5 that is multiply imaged. The mean lens redshift for $z_{S}=5$ is $z_{L}=1.4$. If we select the source from the general galaxy population and the lens from the population of lenses, then the typical source-to-lens surface brightness ratio is $1: 3$ if $\eta=20 \%$ (or close to $1: 1$ if $\eta=2 \%$ ).

Even though lens galaxies might have a somewhat higher mean surface brightness than the sources that they lens, it should be possible to detect lensed sources, since (1) the image center will typically be some distance from the lens center, of the order of half the image separation, and (2) the younger stellar population and higher redshift of the source will make its colors different from those of the lens galaxy, permitting an easy separation of the two in multicolor observations. These two helpful features, along with the fact that the source is much smaller than the lens and the image separation, are evident in the currently known systems that feature galaxy-galaxy lensing. These include two four-image "Einstein cross" gravitational lenses discovered by Ratnatunga et al. (1995) in the Groth-Westphal strip, and a lensed three-image arc detected in the Hubble Deep Field South and studied in detail by Barkana, Blandford, \& Hogg (1999). In these cases of moderate redshifts and optical/UV observations, the sources appear bluer than the lens galaxies.

In the infrared range of NGST, high-redshift sources are generally expected to be redder than their low-redshift lenses, since the overall redshift has a dominant effect on the spectrum. Suppose, e.g., that $z_{S}=5$ and $z_{L}=1.4$. We divide the NGST spectral range into four logarithmically spaced parts (in order of increasing frequency). For a given spectrum, we find the fraction of the total luminosity that is emitted in each frequency quadrant. The mean fractions for $z_{S}=5$ galaxies are $0.37,0.21,0.26$, and 0.16 , respectively, while the fractions for $z_{L}=1.4$ lenses (assuming, as above, that their stars formed at redshift 5) are 0.16, 0.29, 0.39, and 0.16 . Thus, if we use the lowest frequency quadrant, the source will be brighter than the lens by an additional factor of 2.3 relative to the source-to-lens luminosity ratio when we use the full NGST bandwidth. Note that we have not included extinction here, which could further redden the colors of lensed sources.

\section{CONCLUSIONS}

We have calculated the lensing probability of highredshift galaxies or quasars by foreground dark matter halos. We found that the lensing optical depth for multiple imaging of sources increases by a factor of 4-6 from $z_{S}=2$ to $z_{S}=10$. With a magnification bias of $\sim 5$ expected for 
$z_{S}>5$ sources, the fraction of apparent sources that form one of the images of a lensed source reaches $\sim 5 \%$ for sources at $z_{S}=10$ (see Table 1). Among lenses with image separations below $5^{\prime \prime}$, the typical image separation (in $\Lambda \mathrm{CDM}$ ) drops from 1".1 at $z_{S}=2$ to 0.5 at $z_{S}=10$. With its expected $\sim 0$ ".06 resolution, NGST can resolve $\sim 85 \%$ of the lenses with $z_{S}=10$. Assuming the number counts predicted by Haiman \& Loeb (1999), the estimated number of lensed sources above $1 \mathrm{nJy}$ per field of view of NGST is roughly 5 for $z>10$ quasars, 10 for $z>5$ quasars, $1-15$ for $z>10$ galaxies, and 30-200 for $z>5$ galaxies. Note that these values are in a $\Lambda \mathrm{CDM}$ cosmology; the number of $z>10$ galaxies is smaller by a factor of $\sim 3$ in SCDM, but larger by a factor of $\sim 10$ in OCDM.

Although only a small fraction of the sources are multiply imaged, all sources are mildly altered by gravitational lensing due to foreground objects. For a source that is not multiply imaged, the cross section for an amplification of at least $A$ varies as $1 /(A-1)^{2}$ for an SIS lens. Thus, for $z_{S}=10$ the optical depth is unity for an amplification of $A=1.1$ or greater. This implies that extended sources at high redshifts are significantly distorted due to lensing. A typical $z=10$ source is magnified or demagnified by $\sim 10 \%$, and also has an ellipticity of at least $10 \%$ due to lensing.

We have also predicted the size distribution of galactic disks at high redshifts (see Fig. 6) and found that the angular resolution of $N G S T$ will be sufficiently high to avoid confusion noise due to overlapping sources. Indeed, with a $1 \mathrm{nJy}$ flux limit, the probability of encountering a galactic disk inside an aperture of 0.06 diameter is $8.9 \%$ for $\Lambda \mathrm{CDM}$, of which $4 \%$ comes from $z>2$ sources, $1 \%$ comes from $z>5$ sources, and only $0.02 \%$ is contributed by $z>10$ sources (see Fig. 5). These values are for a high star formation efficiency of $\eta=20 \%$, and they are reduced if $\eta=2 \%$.

In our estimates of the lensing rate in $\S 2$, we assumed that a lensed source can be detected even when its images overlap the lensing galaxy. We showed in $\S 3$ that the mean surface brightness of galaxies evolves modestly above redshift 2 (see Fig. 8). Although the surface brightness of a background source will typically be somewhat lower than that of the foreground lens, the lensed images should be detectable, since they are offset from the lens center and their colors are expected to differ from those of the lens galaxy.

Although the typical size of sources decreases with increasing redshift, at least $60 \%$ of the $z>10$ galaxies above $1 \mathrm{nJy}$ can still be resolved by NGST. This implies that the shapes of these high-redshift galaxies can be studied with NGST. We have also found that the high resolution of $N G S T$ is crucial in making the majority of sources on the sky useful for weak-lensing studies.

When we assumed a $1 \mathrm{nJy}$ flux limit for detecting point sources, we included the fact that resolved sources require a higher flux in order to be detected with the same signal-tonoise ratio. Therefore, estimates of number counts that assume a constant flux limit of $1 \mathrm{nJy}$ for all sources overestimate the number counts by a factor of 2 for $z>10$ sources and a star formation efficiency of $\eta=20 \%$, or by as much as a factor of 8 if $\eta=2 \%$. Even with this limitation, however, NGST should detect a total (over all redshifts) of roughly one galaxy per $\operatorname{arcsec}^{2}$ for $\eta=20 \%$ (or one per 4 $\operatorname{arcsec}^{2}$ if $\eta=2 \%$ ).

In conclusion, the field of gravitational lensing is likely to benefit greatly over the next decade from the combination of the unprecedented sensitivity and high angular resolution of NGST.

We thank Zoltan Haiman for providing number count data from earlier work. We are also grateful to Tal Alexander and Amiel Sternberg for numerical results of their stellar population model, and to David Hogg for useful discussions. R. B. acknowledges support from Institute Funds. This work was supported in part by NASA grants NAG 5-7039 and NAG 5-7768 for A. L.

\section{REFERENCES}

Barkana, R., Blandford, R., \& Hogg, D. W. 1999, ApJ, 513, L91 Barkana, R., \& Loeb, A. 1999, ApJ, 523, 54

Brainerd, T. G., Blandford, R. D., \& Smail, I. 1996, ApJ, 466, 623

Bryan, G., \& Norman, M. 1998, ApJ, 495, 80

Cen, R., Gott, J. R., Ostriker, J. P., \& Turner, E. L. 1994, ApJ, 423, 1

Chen, H.-W., Lanzetta, K., \& Pascarelle, S. 1999, Nature, 398, 586

Cheng, Y. N., \& Krauss, L. M. 1999, Int. J. Mod. Phys. A., submitted

Chiba, M., \& Yoshii, Y. 1999, ApJ, 510, 42

Croom, S. M., Shanks, T., Boyle, B. J., Smith, R. J., Miller, L., \& Loaring,

N. S. 1999, in Evolution of Large Scale Structure: From Recombination to Garching, ed A. J. Banday et al. (Enschede: PrintPartners), in press

Dalcanton, J. J., Spergel, D. N., \& Summers, F. J. 1997, ApJ, 482, 659 (DSS)

Dey, A., Spinrad, H., Stern, D., Graham, J. R., \& Chaffee, F. H. 1998, ApJ, 498, L93

Fall, S. M., \& Efstathiou, G. 1980, MNRAS, 193, 189

Fan, X., et al. (SDSS Collaboration). 1999, AJ, 118, 1

Flores, R., Primack, J. R., Blumenthal, G. R., \& Faber, S. M. 1993, ApJ, 412,443

Fukugita, M., Futamase, T., Kasai, M., \& Turner, E. L. 1992, ApJ, 393, 3

Fukugita, M., Hogan, C. J., \& Peebles, P. J. E. 1998, ApJ, 503, 518

Gnedin, N. Y., \& Ostriker, J. P. 1997, ApJ, 486, 581

Gross, M. A. K., Somerville, R. S., Primack, J. R., Holtzman, J., \& Klypin, A. 1998, MNRAS, 301, 81

Haiman, Z., \& Loeb, A. 1997, ApJ, 483, 21 1998, ApJ, 503, 505

. 1999, in After the Dark Ages: When Galaxies Were Young (the Universe at $2<z<5$ ), ed. S. S. Holt \& E. P. Smith (Woodbury: AIP), in press

Hausman, M., \& Ostriker, J. P. 1978, ApJ, 224, 320

Hernquist, L., Spergel, D. N., \& Heyl, J. S. 1993, ApJ, 416, 415

Hoekstra, H., Franx, M., Kuijken, K., \& Squires, G. 1998, ApJ, 504, 636

Hu, E. M., Cowie, L. L., \& McMahon, R. G. 1998, ApJ, 502, L99
Hudson, M. J., Gwyn, S. D. J., Dahle, H., \& Kaiser, N. 1998, ApJ, 503, 531

Kepner, J. V., Babul, A., \& Spergel, D. N. 1997, ApJ, 487, 61

Kochanek, C. S. 1995, ApJ, 453, 545 . 1996a, ApJ, 466, 638 .1996b, ApJ, 473, 595

Kravtsov, A. V., Klypin, A. A., Bullock, J. S., \& Primack, J. R. 1998, ApJ, 502,48

Lacey, C. G., \& Cole, S. M. 1993, MNRAS, 262, 627

Loeb, A., \& Haiman, Z. 1997, ApJ, 490, 571

Luppino, G., \& Kaiser, N. 1997, ApJ, 475, 20

Madau, P. 1999, in Phys. Scr., in press (preprint astro-ph/9902228)

Madau, P., Haardt, F., \& Rees, M. J. 1999, ApJ, 514, 648

Marri, S., \& Ferrara, A. 1998, ApJ, 509, 43

Mo, H. J., Mao, S., \& White, S. D. M. 1998, MNRAS, 295, 319 (MMW)

Moore, B., Quinn, T., Governato, F., Stadel, J., \& Lake, G. 1999, MNRAS, submitted (preprint astro-ph/9903164)

Nakamura, T. T., \& Suto, Y. 1997, Prog. Theor. Phys., 97, 49

Narayan, R., \& White, S. D. M. 1988, MNRAS, 231, 97P

Navarro, J. F., Frenk, C. S., \& White, S. D. M. 1997, ApJ, 490, 493 (NFW)

Navarro, J. F., \& Steinmetz, M. 1997, ApJ, 478, 13

Peebles, J. P. E. 1993, Principles of Physical Cosmology (Princeton: Princeton Univ. Press)

Pen, U.-L. 1998, ApJ, 498, 60

Porciani, C., \& Madau, P. 2000, ApJ, 532, in press (preprint astro-ph/ 9810403)

Press, W. H., \& Schechter, P. 1974, ApJ, 187, 425 (PS)

Quinn, T., Katz, N., \& Efstathiou, G. 1996, MNRAS, 278, L49

Ratnatunga, K. U., Ostrander, E. J., Griffiths, R. E. \& Im, M. 1995, ApJ, 453, L5

Schneider, P., \& Kneib, J.-P. 1998, in The Next Generation Space Telescope: Science Drivers and Technological Challenges (ESA SP 429; Noordwijk: ESA), 98 
Seitz, C., Kneib, J.P., Schneider, P \& Seitz, S. 1996, A\&A, 314, 707

Sellwood, J. A., \& Moore, E. M. 1999, ApJ, 510, 125

Spinrad, H., Stern, D., Bunker, A., Dey, A., Lanzetta, K., Yahil, A., Pascarelle, S., \& Fernández-Soto, A. 1998, AJ, 116, 2617

Sternberg, A. 1998, ApJ, 506, 721

Thomas, D., \& Kauffmann, G. 1999, PASP, in press (preprint astro-ph/ 9906216)

Thoul, A. A., \& Weinberg, D. H. 1996, ApJ, 465, 608

Turner, E., Ostriker, J. P., \& Gott, J. R., III. 1984, ApJ, 284, 1
Wambsganss, J., Cen, R., \& Ostriker, J. P. 1998, ApJ, 494, 29

Wambsganss, J., Cen, R., \& Ostriker, J. P., \& Turner, E. L. 1995, Science, 268,274

Warren, M. S., Quinn, P. J., Salmon, J. K., \& Zurek, W. H. 1992, ApJ, 399, 405

Weinberg, D. H., Hernquist, L., \& Katz, N. 1997, ApJ, 477, 8

Weymann, R. J., Stern, D., Bunker, A., Spinrad, H., Chaffee, F. H., Thompson, R. I., \& Storrie-Lombardi, L. J. 1998, ApJ, 505, L95 